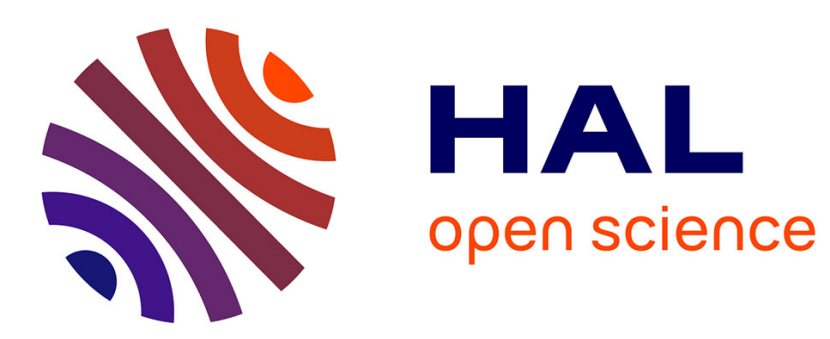

\title{
Optimization of the predefined number of replications in a Ultra Narrow Band based IoT network
}

Yuqi Mo, Minh-Tien Do, Claire Goursaud, Jean-Marie Gorce

\section{To cite this version:}

Yuqi Mo, Minh-Tien Do, Claire Goursaud, Jean-Marie Gorce. Optimization of the predefined number of replications in a Ultra Narrow Band based IoT network. Wireless Day, Mar 2016, Toulouse, France. pp.1 - 6, 10.1109/WD.2016.7461514 . hal-01389335

\section{HAL Id: hal-01389335 \\ https://inria.hal.science/hal-01389335}

Submitted on 28 Oct 2016

HAL is a multi-disciplinary open access archive for the deposit and dissemination of scientific research documents, whether they are published or not. The documents may come from teaching and research institutions in France or abroad, or from public or private research centers.
L'archive ouverte pluridisciplinaire HAL, est destinée au dépôt et à la diffusion de documents scientifiques de niveau recherche, publiés ou non, émanant des établissements d'enseignement et de recherche français ou étrangers, des laboratoires publics ou privés. 


\title{
Optimization of the predefined number of replications in a Ultra Narrow Band based IoT network
}

\author{
Yuqi MO*†, Minh-Tien DO*†, Claire GOURSAUD*, and Jean-Marie GORCE* \\ *CITI Labs, INSA-Lyon, Villeurbanne, France \\ $\dagger$ Sigfox Company, Building E-volution, Labège, France \\ Email:mo.yuqi@insa-lyon.fr; minhtien.do@sigfox.com; claire.goursaud@insa-lyon.fr; \\ jean-marie.gorce@insa-lyon.fr;
}

\begin{abstract}
Ultra Narrow Band networks using RandomFTMA scheme are mainly characterized by the randomness both in time and frequency domain. The frequency randomness prevents from allocating orthogonal resources for transmission, and induces uncontrolled interference. By introducing diversity, replication mechanism is a promising candidate to enhance the reliability of such wireless network. Therefore, in this paper, by taking into account the randomness both in time and frequency domain, the replication mechanism has been considered. Considering the outage probability, we theoretically evaluate the system performance and show that there exists an optimal number of transmissions. Finally, we highlight that this number of repetitions can be easily optimized by considering a unique global parameter.
\end{abstract}

Keywords-Wireless sensor network; UNB transmission; Random-FTMA schemes, replication mechanism.

\section{INTRODUCTION}

IoT (Internet of things) networks are gaining more and more interest as they will provide connectivity for countless number of devices [1]-[3]. When the goal is to collect information (for example, for temperature monitoring, parking availability monitoring, smart metering, ...), the devices will require to transmit only a few amount of data, during a small proportion of time. In this case, the base station should cover as much devices as possible to reduce the cost of infrastructure deployment, and not waste transmission resources. This is the reason why long range technologies such as LoRa [5] and Ultra Narrow Band (UNB) [6] have emerged recently. The main advantage of UNB over LoRa is that it does not rely on access protocol to handle multiple access [4]. Thus, the transmission resources needed for channel reservation are saved.

Nevertheless, UNB suffers from interference that may lead to the loss of some transmitted packets. Indeed, in SigFox UNB based network, devices access to the medium at will. Transmissions are seen as random, both in time and frequency (R-FTMA : Random Frequency and Time Multiple Access), due to the non-negligible jitter impact [7]. This induces some simultaneous transmission in the same frequency area, leading to interference. The system performance of such network was evaluated in [8], and the capacity in term of the devices number was estimated. However, no control is made on the transmission, so when the interference is too high the packet information is definitively lost.
In the literature [9], the reliability of data delivery can be achieved by two ways: retransmission-based mechanism and redundancy-based mechanism. The basic principle of the retransmission schemes is to repeatedly transmit the failed message which cannot be recovered at the receiver. The demand of replication happens until a correct message arrives. To know that the message is correctly received or not between the transmitter and receiver, an acknowledgment mechanism must be applied. However, this kind of protocol requires a downlink transmission, so the reservation of transmission resources. This might further aggravate the inherent congestion of the medium.

Besides, in the redundancy-based mechanism, multiple copies of the same message are transmitted based on Erasures Coding that allows a receiver to recover the information from independent message loss. The popular types of Erasure Coding are Reed-Solomon codes, lowdensity parity-check (LDPC) codes, Fountain Codes, and the simplest one is the replication mechanism.

The performance of retransmission and redundancybased mechanism is theoretically evaluated in [10] in term of the energy efficiency. Indeed, both retransmission-based mechanism and redundancy-based mechanism are able to improve the data delivery reliability, but also consume a lot of battery-energy of a sensor node which is inherently limited. Besides, this theoretical analysis highlights that Erasure Coding mechanism is out-performing compared to the replication mechanism in term of reliability and energy efficiency, for the case of a low packet loss rate and low number of hops.

In the UNB network using Random-FTMA schemes, the one-hop communication between active node and basestation is essential to reduce the energy consumption. We thus consider the replication redundancy-based mechanism in this paper. We consider only the transmission reliability in terms of the outage probability according to the number of replications. To this aim, the coding, channel and the efficient consumption of battery energy is neglected in our study for the sake of simplicity. Based on the PHY analysis (SINR: Signal to Interference plus Noise Ratio), we define that the message failure occurs when the BER calculated at the base-station is less than the BER threshold (typically $10^{-3}$ or $2 \cdot 10^{-4}$ for BPSK modulation). Each node transmits its message to basestation with a predefined number of occurrences regardless of the energy consumption, and the success state of the 
previous transmissions. One may expect that the repetition increases the chances of receiving at least a copy correctly. But, it also increases the load, thus, the probability of collisions, leading to a compromise. Such phenomenon has already been studied for classical transmissions [11][13], but, to the best of the authors' knowledge, not for UNB transmissions where the frequency multiple access is specific. In this paper, we thus aim at finding the optimal number of replications for such network.

The rest of this paper is organized as follows. The assumptions and modeling are detailed in Section II. Next, we theoretically evaluate the system performance for the simple replication scheme in Section III. Then, we exploit the obtained formulas to estimate the optimum replication number in Section IV. Finally, Section V gives the conclusion.

\section{Modeling And Assumption}

\section{A. Network Topology}

In this study, the network is reduced to a unique basestation (BS) covering a large number of nodes positioned in this coverage. We consider that all the nodes are received at the base station with the same power. This corresponds to the case with a power control loop.

In this study, we consider a point in time where $N$ nodes are active. At a given time, only a few portion of these $N$ nodes are actually transmitting. The sleep or wake-up duty cycle may vary among the different nodes. Each node randomly chooses its transmission time slot.

More specifically to UNB, the choice of carrier frequency is performed independently in each node without any prior knowledge on the occupation of the channel and can be discrete or continuous [7]. At the base station, the whole transmission band $B W$ is analyzed. When a transmission is detected, the received signal is filtered at the corresponding frequency before demodulation. In case of simultaneous transmission, the interference depends on the spacing $\delta_{f}=\left|f_{1}-f_{2}\right|$ between the desired node frequency $f_{1}$ and the interferer's one $f_{2}$ :

$$
P_{I}(t)=P\left(\delta_{f}, t\right)
$$

The interference level for a realistic UNB filter shape is plotted on Fig. 1. We can observe that the interference level is low if the frequency spacing $\delta_{f}$ is high. Thus, a unique interferer leads to failure if $\delta_{f}$ is small.

The system performance is evaluated through the outage probability (OP). A data transmission is considered successful if the received SINR is above a predefined threshold $\gamma^{*}$, with :

$$
\mathrm{SINR}=\frac{P_{s}}{\left(W+P_{I}\right)}
$$

where $W$ is the filtered additive white Gaussian noise power assumed to be negligible compared to the interference level, which is a reasonable value for UNB systems. We consider $\gamma^{*}=8 \mathrm{~dB}$ (corresponding to the Bit-ErrorRate $2 \cdot 10^{-4}$ for our numerical results as we consider the BPSK modulation. We have determined that, with the
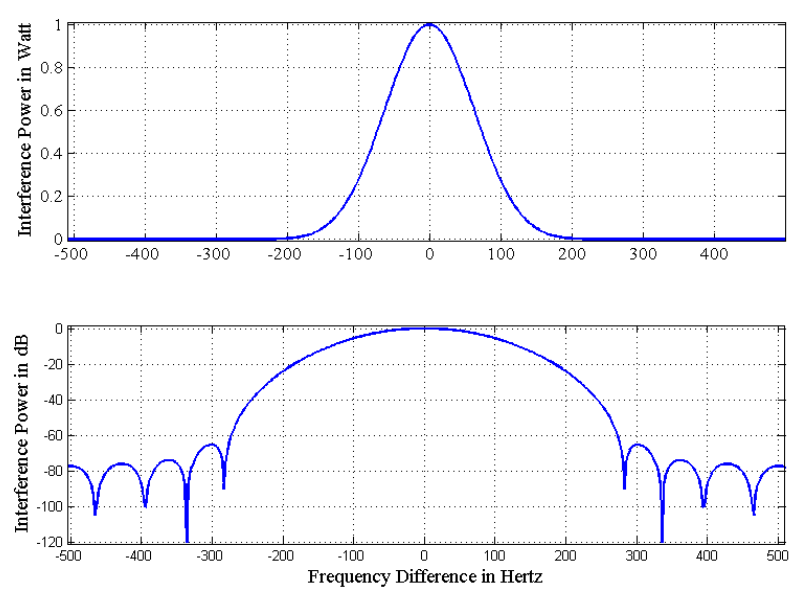

Figure 1. Interference behavior $P_{I}$ as a function of the frequency spacing $\delta_{f}$ between desired user and interferer.

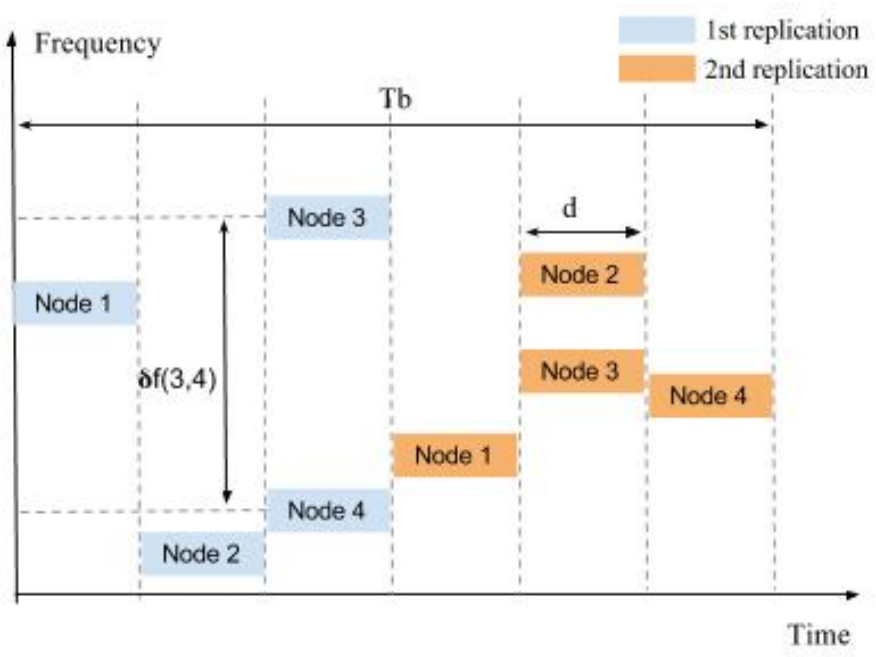

Figure 2. Illustration of replication $n_{r}=2$ for duration of message $d$, period of message $T b$

considered filter shape, the BER $<2 \cdot 10^{-4}$ is obtained for $\delta_{f}>123 \mathrm{~Hz}$ ).

\section{B. Replication Mechanism}

Any active node $i \in[1, \cdots, N]$ randomly selects a carrier $f_{i}$ and a time slot $t_{i}$. Failure occurs when 2 active nodes (a desired node $x$ and interfering node $y$ ) transmit simultaneously, and the frequency shift between their carriers $\delta_{f}=\left|f_{x}-f_{y}\right|$ falls in the interval $[0 ; 123]$ $\mathrm{Hz}$.

To improve the service probability for each transmitting node, the replication mechanism illustrated in Figure 2 is applied. We assume that each message (with transmission duration $d$ ) should be transmitted in less than the message lifetime $T_{b}$. As the system is considered slotted, there are $\frac{T_{b}}{d}$ slots available for transmission during the message lifetime. We consider that the message is transmitted exactly $n_{r}$ times during the message lifetime $T_{b}$, regardless of the success of previous transmissions. $n_{r}$ is thus considered 
predefined and identical for all nodes. The length of the window allocated for a replication is thus $\frac{T_{b}}{n_{r} \cdot d}$. Each node randomly selects a time slot in this window to repeat its message. The message is successfully transmitted when at least 1 of the $n_{r}$ attempts succeeds. Otherwise, the message is considered lost.

\section{TheORETICAL ANALYSIS}

\section{A. Outage Probability derivation}

In this section, we derive a theoretical expression for the outage probability, as a function of the main system parameters : the whole transmission band $B W$; the frequency occupancy with respect to the carrier $[-b ; b] \mathrm{Hz}$; the wake-up duty cycle of nodes $T_{b}$; the time duration of a message $d$, and the replication number $n_{r}$. The first four factors influence the channel occupancy of each replication in time and frequency domains.

The goal of the base station is to decode all transmitted messages, from all the users. However, without sake of generality, in this analysis, we consider the transmission status of a unique user, called "desired user". We evaluate its probability to have its message lost, due to other users called "interferers".

We first consider the case where the message is transmitted with a single replication. A node wakes up every $T_{b}$ seconds (one may note that the time unit can also be minute, hour, ...) to send a message who lasts $d$ seconds. Thus, for time-slotted multiple access, with slot duration $d$, the probability that a given potential interferer transmits during the same slot as the desired user can be expressed by :

$$
P t=\frac{d}{T_{b}}
$$

The same principle applies for the frequency domain. In this theoretical analysis, to simplify the expression, we suppose that transmission errors are due to a unique interferer. We neglect the cases where 2 or more users jointly generate an interference level that leads to an error. Therefore, with this assumption, there is an error if an interferer elected a carrier frequency spaced by less than $b$ compared to the desired user's one. Thus, the frequency interfering zone that causes a failure if the frequency gap of non-desired node and the desired node has a width $2 b$. We can deduce that the frequency collision probability is:

$$
P f=\frac{2 b}{B W}
$$

A collision occurs when two nodes send their messages at the same time, and when their frequency spacing is too small. As $P f$ and $P t$ are independent, the collision probability is $P_{t} \cdot P_{f}=\frac{2 b \times d}{B W \times T_{b}}$.

As a consequence, when considering $N$ independent potential users, the probability for a given user message being correctly received, without considering replication mechanism, is the probability that none of the $N-1$ other user creates collision:

$$
P\left(C=0 \mid n_{r}=1\right)=\left(1-\frac{2 b \times d}{B W \times T_{b}}\right)^{N-1}
$$

with $C$ the number of collisions, and $n_{r}$ the number of replications.

We now extend this expression to the case with replication mechanism. First, we modify (5) to take into account the fact that the number of transmissions increases. Indeed, the collision probability of a replication with any replication of another user is multiplied by $n_{r}$ and becomes $\frac{2 b \times d}{B W \times T b} \times n_{r}$

Thus, the probability that a given replication is correctly received can be expressed as:

$$
P\left(C=0 \mid n_{r}\right)=\left(1-\frac{2 b \times d}{B W \times T_{b}} \times n_{r}\right)^{N-1}
$$

As replications are identical, the message is successfully received if at least one of this replication is correctly received at the base station. We make the assumption that the replications experience independent transmission conditions, and that the success probability of any replication is independent of the collision on the previous ones. Accordingly, the outage probability of one useful message, transmitted with $n_{r}$ replication, is:

$$
\mathrm{OP}=\left(1-\left(1-\frac{2 b \times d}{B W \times T_{b}} \times n_{r}\right)^{N-1}\right)^{n_{r}}
$$

One may note that, in practice, the collision probability of a given replication depends on the previous replications state. Indeed, a given replication of an interferer can collide with at most one replication of the desired user. Thus, the collision probability should be updated for each replication, depending on the number of collisions observed during the previous replications. However, as the number of users (thus the number of transmissions) is very high, the collision probability decrease can be neglected.

\section{B. Validation}

In this section, we validate by simulation the theoretical OP established in eq.7. We consider the impact of 4 parameters : $B W, T_{b}, n_{r}$ and $N$. We do not consider $b$ variation as this parameters is constrained by the transmission technology. Furthermore, as the system is slotted, the exact values of $d$ and $T_{b}$ are not of importance, but rather the number of available slots for each message: $\frac{T_{b}}{d}$. Thus, we fix $d=1$, and only vary $T_{b}$.

We have plotted on Figures 3-5, the comparison of the theoretical OP with the simulated one, as a function of the replication numbers, when varying $N, B W$, or $T_{b}$. We can first observe that the curves does not match perfectly, but theory still provides a good estimation of the observed OP, especially for low $n_{r}$. More importantly, the theory correctly describes the evolution of the OP as a function of $n_{r}$

Besides, these figures highlight the fact that the replication mechanism is able to greatly improve the probability of successful transmission of a message. Indeed, the OP can be decreased compared to the one replication case. This is due to the fact that the increased number of replications $n_{r}$ initially permits to increase the chance 


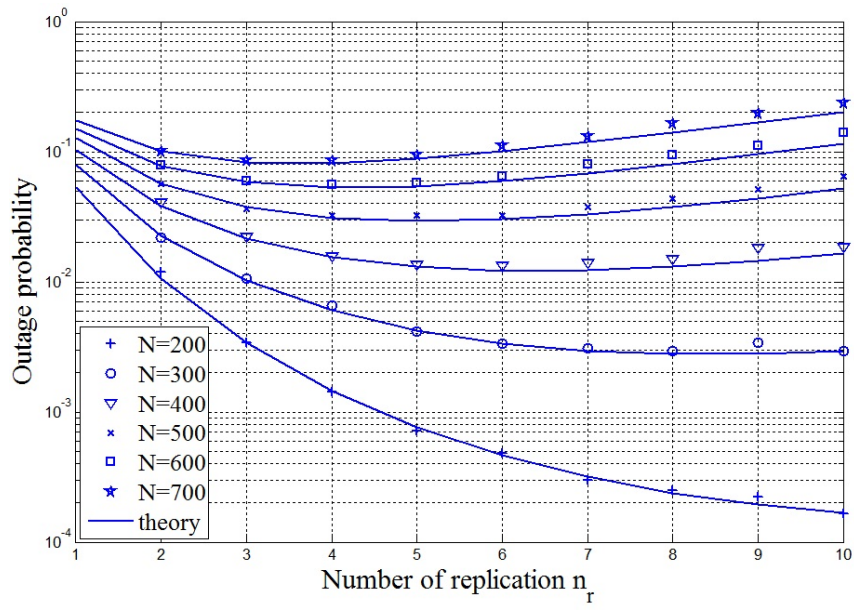

Figure 3. OP vs number of replications $n_{r}$ with different number of active users $N, B W=12 \mathrm{kHz}, T_{b}=75 \mathrm{~s}$.

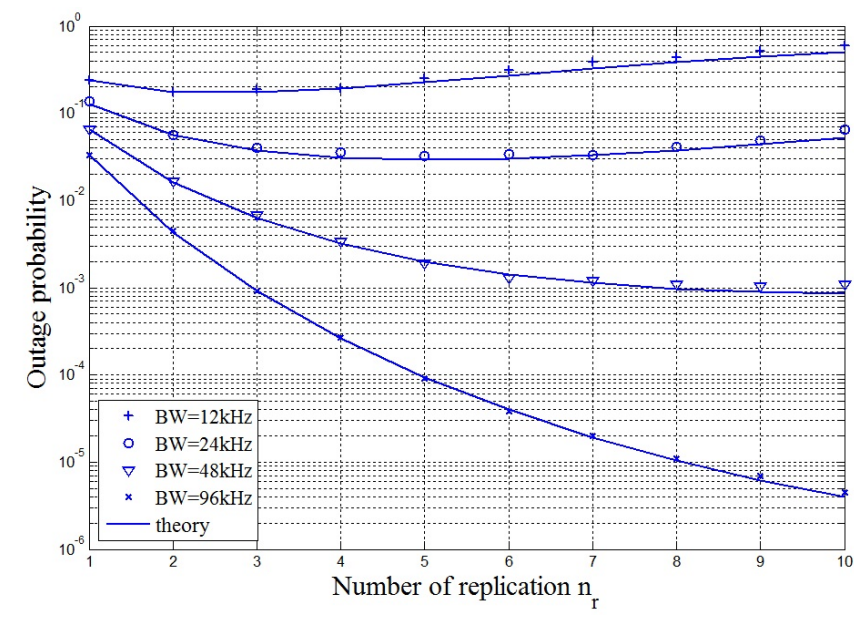

Figure 4. OP vs number of replications $n_{r}$ with different bandwidth $B W, T_{b}=75 s, N=1000$.

that at least one packet is successfully received. However, the repetition also multiplies the number of messages sent by the nodes within a fixed time interval, and thus increases the channel utilization and the collision probability. Therefore, for a large number of replication $n_{r}$, the temporal and frequency resources become overloaded, and the benefit of adding redundancy is disabled by the increase of interference level. Thus the replication process is both increasing the collision probability (thus the message error probability), while also increasing the chance to have at least one replication successfully received (thus decreasing the message error probability). Consequently, we can observe on Figure 3-5, that there is an optimum number of replications, that permits to obtain the highest message throughput.

As the theory evolution is consistent with the simulated one, we use from now on eq.7 to determine the optimum number of replications.

\section{REPLICATION NUMBER OPTIMIZATION}

In this section, we focus on the number of replications that permits to get the lowest OP.

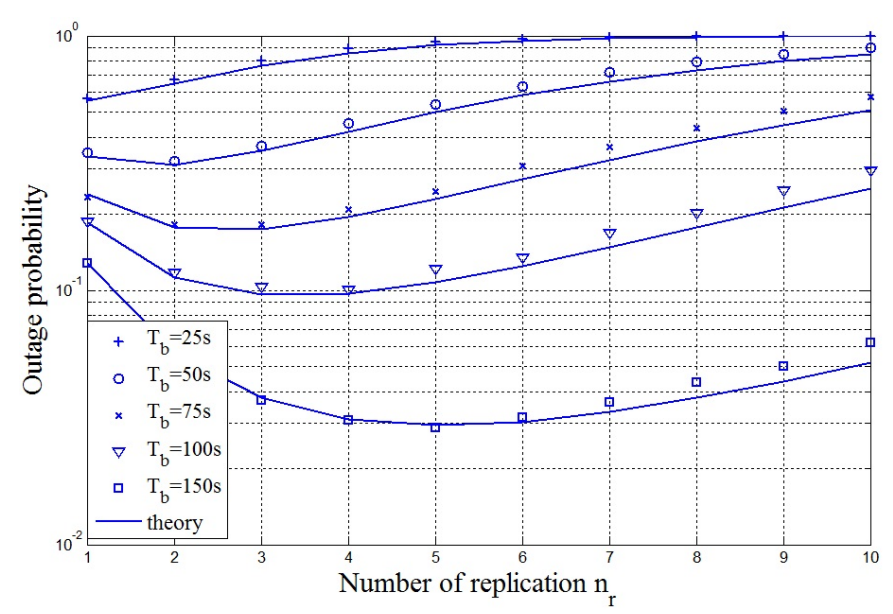

Figure 5. OP vs number of replications $n_{r}$ with different message period $T_{b}, B W=12 \mathrm{kHz}, N=1000$.

\section{A. Preliminary estimation of $n_{r_{o p t}}$}

OP is influenced by many parameters. For sake of simplification, we call $\lambda$ the time-frequency collision factor, with:

$$
\lambda=\frac{2 b \times d}{B W \times T b}
$$

Hence OP simplifies to:

$$
\mathrm{OP}=\left(1-\left(1-\lambda \times n_{r}\right)^{N-1}\right)^{n_{r}}
$$

We derive eq. 9 with respect to $n_{r}$ to find out $n_{r_{o p t}}$.

$$
\begin{aligned}
\frac{\mathrm{d} O P}{\mathrm{~d} n_{r}}= & \left(\frac{(N-1) \lambda n_{r}\left(1-\lambda n_{r}\right)^{N-2}}{1-\left(1-\lambda n_{r}\right)^{N-1}}\right. \\
& \left.\quad+\ln \left(1-\left(1-\lambda n_{r}\right)^{N-1}\right)\right) \\
& \times\left(1-\left(1-\lambda n_{r}\right)^{N-1}\right)^{n_{r}}
\end{aligned}
$$

As OP is convex, $n_{r_{o p t}}$ is obtained when $\frac{\mathrm{d} O P}{\mathrm{~d} n_{r}}=0$. We do not consider the case where $\left(1-\left(1-\lambda n_{r}\right)^{N-1}\right)^{n_{r}}$ is equal to 0 , as this would lead to a null OP. So the expression of $n_{r_{o p t}}$ can be deduced from the first term of the multiplication.

However, $n_{r_{o p t}}$ can not be solved analytically. Nonetheless, its value can be numerically evaluated, depending on the values of $\lambda$ and $N$.

We have plotted on Figure 6 the evolution of $n_{r_{o p t}}$ when considering different time-frequency factor $\lambda$ and number of active users $N$. This figure shows that when the time-frequency factor $\lambda$ increases, the optimal number of replication $n_{r_{o p t}}$ decreases. This means that when collision probability is already important, the replication mechanism make transmission even more difficult. On the contrary, for low $\lambda$, the replication mechanism permits to take advantage of the under-used channel to improve the transmission quality. Similarly, for a given $\lambda$, the number 


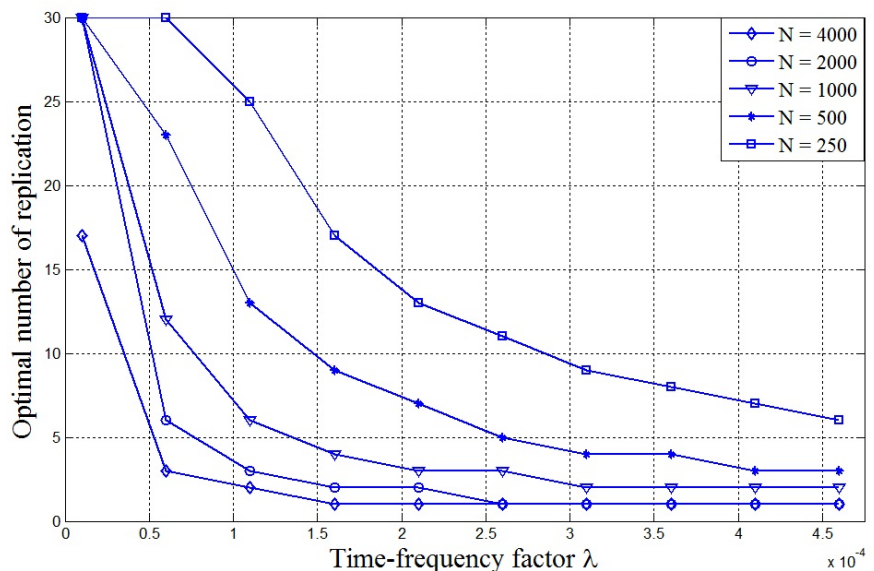

Figure 6. Optimal replication $n_{r_{\text {opt }}}$ vs time-frequency factor $\lambda$ and active user numbers $N$

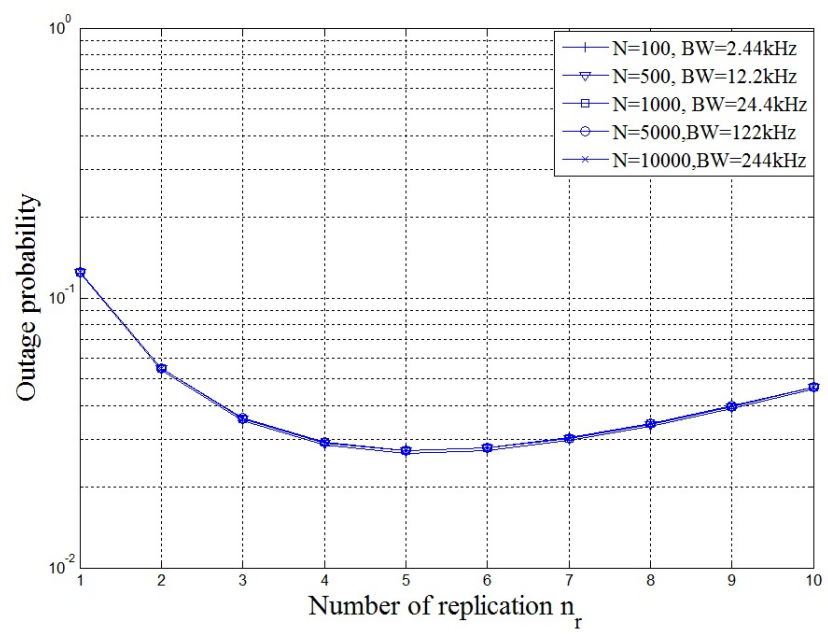

Figure 7. OP vs number of replications $n_{r}$ with $\frac{N}{B W}=0.04, T_{b}=75$ s.

of replications should be decreased as the number of active users increases.

Thus, from this theoretical analysis, we can confirm that the optimal number of replication decreases when the network is overloaded, which could be caused by : too many active users at the same time; users sending message too often; or a small available bandwidth.

\section{B. Parameters simplification}

We can further our analysis by evaluating OP when considering a constant repartition of users in time or frequency. To do so, we have computed eq. 7 for special cases of $\frac{N}{B W}=$ cste and $\frac{N}{T b}=$ cste, and plotted the results on Figure 7 and Figure 8.

As shown in Figure 7 and Figure 8, we can observe that when the parameters $B W$ and $T_{b}$ evolve proportionally to $N$, the behavior of the OP according to the number of replications $n_{r}$ is unchanged. Therefore, it is possible to simplify our study by reducing the number of parameters from 3 (i.e., $B W, T_{b}$ and $N$ ) to 2 (i.e., $\frac{N}{B W}=$ cste and $\frac{N}{T_{b}}=$ cste). But, by jointly exploiting the above properties, we can further simplify to a single parameter :

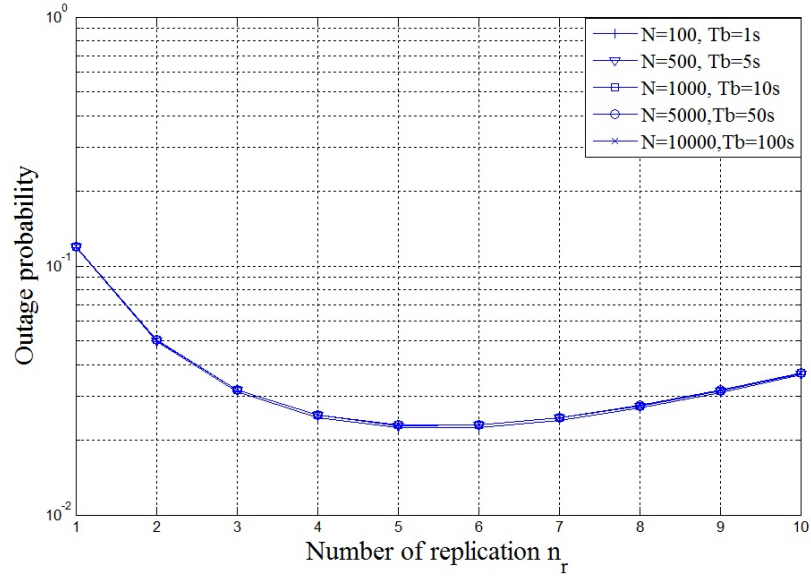

Figure 8. OP vs number of replications $n_{r}$ with $B W=192 \mathrm{kHz}$, $\frac{N}{T_{b}}=100$.

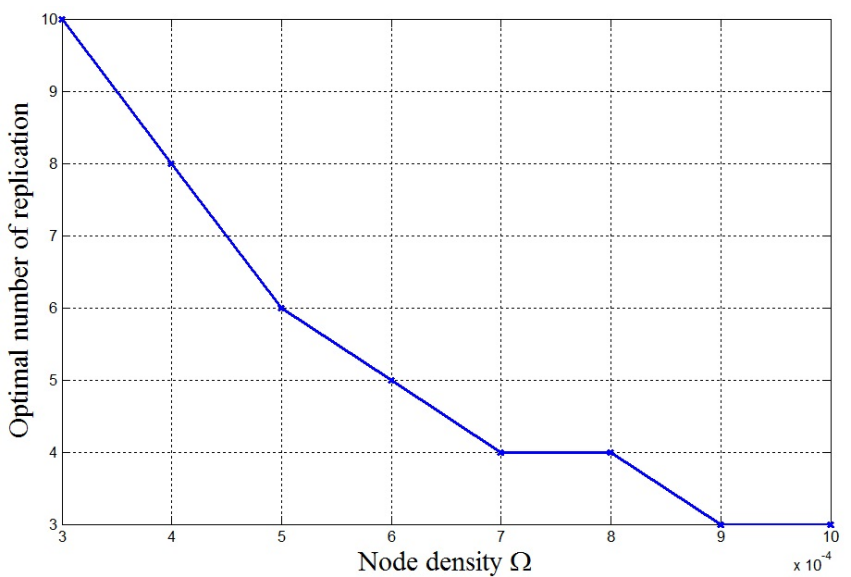

Figure 9. Node density $\Omega$ vs optimal number of replications $n_{r_{o p t}}$.

node density on the temporal and frequency resources $\Omega$, defined as the ratio between the number of nodes $N$ and the temporal and frequency resources $\Omega=\frac{N}{B W \times T_{b}}$.

From the previously tested sets, we can extract the value of the optimal number of replications $n_{r_{o p t}}$ for different node density $\Omega$. It may be noted that obtained couples $\left(n_{r_{o p t}}, \Omega\right)$ correspond to optimal values of different OP. The evolution of the optimal number of replications $n_{r_{o p t}}$ according to user density $\Omega$ is plotted in Figure 9. We can observe that, $n_{r_{o p t}}$ decreases when the node density gets high (this is due to the fact that the increase of the network load reduces the available time/frequency resources for the replication process). This result is very interesting and promising because its urges the potential ability of configuring the network by relying on a single global parameter: node density over the temporal and frequency resources $\Omega$.

\section{CONCLUSION}

In this paper, we have considered a UNB based network using Random-FTMA scheme. We have proposed and studied a repetition mechanism to improve the reliability of the transmissions. The outage probability is theoreti- 
cally derived and evaluated under different scenarios with parameters such as the number of replications $n_{r}$, the number of nodes $N$, the bandwidth length $B W$ and the message lifetime $T_{b}$. We have shown that the outage probability can be evaluated by considering, in addition to $n_{r}$ a single criteria : the node density over the temporal and frequency resources $\Omega$. Besides, we have highlighted that, for each node density $\Omega$, there is an optimal number of replications $n_{r_{\text {opt }}}$, which we have estimated. With such results, the number of replications can be easily predefined and adapted to the network load. Finally, one may note that this study can be furthered by estimating the minimal number of replications needed to obtain a targeted OP.

\section{ACKNOWLEDGMENT}

The authors would like to thank Christophe Fourtet (Sigfox, Toulouse) for his helpful inputs regarding the UNB parameters during this study.

\section{REFERENCES}

[1] I. Ishaq, D. Carels, G.K. Teklemariam, J. Hoebeke, F.V.D. Abeele, E.D. Poorter, and P. Demeester (2013). "IETF standardization in the field of the internet of things (IoT): a survey“", in Journal of Sensor and Actuator Networks, 2(2), 2013, pp235-287.

[2] L. Da Xu, W. He, S. Li, "Internet of Things in industries: A survey" in IEEE Transactions on Industrial Informatics, 10(4), 2014, pp2233-2243.

[3] A. Zanella, N. Bui, A. Castellani, L. Vangelista and M. Zorzi "Internet of Things for smart cities", IEEE Internet Things J., vol. 1, no. 1, pp.22 -32 2014

[4] C. Goursaud and J. M. Gorce, "Dedicated networks for IoT: PHY / MAC state of the art and challenges", EAI Endorsed Transactions on Internet of Things, vol. 15, no. 1, October 2015

[5] http://lora-alliance.org/What-Is-LoRa/Technology (accessed 2015/07/01)

[6] http://www.sigfox.com/en/\#!/technology 2015/07/01)

(accessed

[7] M.-T. Do, C. Goursaud, and J.-M. Gorce, "On the benefits of Random-FDMA schemes in ultra narrow band networks," in Modeling and Optimization in Mobile, Ad Hoc, and Wireless Networks (WiOpt), 2014 12th International Symposium on, pp. 672-677, May 2014.

[8] M.-T. Do, C. Goursaud, and J.-M. Gorce, "Interference modelling and analysis of random fdma schemes in ultra narrowband networks," in The Tenth Advanced International Conference on Telecommunications, AICT 2014, pp. 132137, July 2014.

[9] M. A. Mahmood, W. K. Seah, and I. Welch, "Reliability in wireless sensor networks: A survey and challenges ahead," Computer Networks, vol. 79, no. 0, pp. 166 187, 2015.

[10] H. Wen, C. Lin, F. Ren, Y. Yue, and X. Huang, "replication or redundancy: Transmission reliability in wireless sensor networks," in Mobile Adhoc and Sensor Systems, 2007. MASS 2007. IEEE International Conference on, pp. 17, Oct 2007.
[11] X. Liu and H. Zhu, "Novel packet Retransmission in ofdma systems using frequency diversity," in Vehicular Technology Conference (VTC Spring), 2011 IEEE 73rd, pp. 15, May 2011.

[12] L. Tong, Q. Zhao, and G. Mergen, Multipacket reception in random access wireless networks: From signal processing to optimal medium access control, IEEE Commun. Mag. , pp. 108112, Nov. 2001

[13] R.S. Sudhaakar, S. Yoon, J. Zhao and C. Qiao, "A Novel QoS-Aware MAC Scheme Using Optimal Retransmission for Wireless Networks", in IEEE Trans. Wireless Comm., vol. 8, no. 5, pp. 2230-2235, May 2009 